\title{
S-9-12 Hydroxyl Radical Generation from Heart Mitochondria Damaged by Ischemia
}

\author{
R.OGURA
}

Department of Medical Biochemistry, Kurume University School of Medicine, 67, Asahi-machi, Kurume 830 (Japan)

\begin{abstract}
I. INTRODUCTION
Metabolism of heart tissue in ischemic injury has been principal interest for many years. Recent evidence suggests that oxygen-derived free radicals may be abundantly produced in ischemic tissues, accounting for at least part of the damage that results. A number of studies have been done to clarify the role of active oxygen species generated from mitochondria $[1,2,3,4]$. It is of interest to know what kind of oxygen free radicals are generated in the ischemic heart, especially in mitochondria. We used an electron spin resonance (ESR) technique with spin trapping to detect the generation of oxygen free radicals from mitochondria isolated from ischemic myocardium.
\end{abstract}

II. PREPARATION OF MITOCHONDRIA FROM GLOBAL ISCHEMIA

The hearts isolated from normal rats were perfused by noncirculating Langendorff method at a constant pressure of $80 \mathrm{~cm}$ in water, with a Krebs-Henseleit buffer solution, which was bubbled with 95\% oxygen and 5\% carbon dioxide gas. A 30 min period of control perfusion was allowed for the hearts, to reach a stable left ventricular function. Subsequently, the hearts were subjected to 60 min of global ischemia. Following the $60 \mathrm{~min}$ ischemia, the mitochondria were prepared from the hearts by differential centrifugation $[5,6,7]$.

III. ESR EXPERIMENT ON THE ISOLATED HEART MITOCHONDRIA

The generation of oxygen free radicals from mitochondria was examined after the addition of respiratory substrate, succinate or glutamate \& malate. For detecting the generation of oxygen radicals from mitochondria, we used an ESR technique with spin trapping $[6,7,8]$. The spin trap agent is DMPO, 5-5'dimethyl-1-pyrroline-N-oxide. The recording of the ESR spectrum started exactly 1 min after the addition of succinate to the reaction mixture. The ESR spectra were recorded on a JEOL $\mathrm{X}$-band spectrometer, model JES-3X.

Fig.1 shows the ESR spectra of DMPO spin adduct obtained from intact heart mitochondria. The upper panel indicates the ESR spectrum obtained from mitochondria incubated with succinate alone. There is no significant characteristic signal. However, after the addition of antimycin A, which is an electron transfer inhibitor at the site-II, the ESR spectrum shows remarkable peaks. From this ESR spectrum, the spin adduct was assigned as the DMPO-hydroxyl radical adduct, by a quartet signal with hyperfine splitting; aN and aH are $15.0 \mathrm{G}$. It was suggested that heart mitochondria release a small amount of hydroxide radicals in the study-state. It was confirmed that the DMPo-hydroxyl radical adduct is not derived from the transition of DMPO-superoxide anion adduct, but derived from the hydroxyl radical generated directly from mitochondria, by using addition of a hydroxyl radical scavenger $[7,9]$ such as ethanol.

Fig.2 shows the ESR spectra of DMPO spin adduct in mitochondria isolated from 
60 min global ischemic heart muscle. A remarkable enhancement of the signal peak is observed. It is suggested that the enhanced generation of hydroxyl radicals in ischemic myocardium leads to the severe oxygen toxicity as seen in ischemic patients.

Next comes the question of what site in the electron transport system generates hydroxyl radicals. Fig.3 indicates the values for hydroxyl radical formation when mitochondria are incubated with succinate and an electron transport inhibitor, either antimycin A or sodium azide. The highest values for hydroxyl radicals formation is in the presence of antimycin $A$, and that they are lower in the presence of sodium azide.

In the case of the glutamate-malate as substrate (Fig.4), a similar pattern was observed. The highest values appeared in the presence of antimycin A, they were less in sodium azide, and much less in rotenone. From the experiments in which an inhibitor was used, the site II of the Col-cytochrome $C$ area, is responsible for the release of electrons, leading to the generation of active oxygen species.

The origin of the hydroxyl radicals which appeared in mitochondria was determined. The generation of active oxygen from the inner membrane of mitochondria (submitochondrial particles) instead of the whole mitochondria was examined. As shown in Fig.5, the ESR spectrum of the spin adduct in submitochondrial particles indicates a spectrum corresponding to that of the superoxide anion adduct [6]. It is of interest that the ESR spectrum of the DMPOsuperoxide anion adduct obtained from ischemic muscle was enhanced remarkably, 1.5 times higher than that of the normal heart. In addition, the superoxide dismutase (SOD) activity in the matrix of mitochondria isolated from ischemic heart muscle was reduced remarkably from 71.9 units to 53.8 units.

The remarkable generation of superoxide anion in submitochondrial particles is consistent with the high generation of hydroxy radicals appearing in whole mitochondria.

Next comes the process of generating hydroxyl radicals from superoxide anion in mitochondrial matrix. SOD and catalase were added to the mitochondrial suspension to find out what would happen to the generation of hydroxyl radicals from mitochondria. The generation of hydroxyl radicals was inhibited by the addition of SOD or catalase. By the addition of catalase at a high concentration $(5,000$ unit per $\mathrm{ml})$, the spectrum of DMPO-hydroxyl radical adduct disappeared completely. From these results, a generation mechanism of hydroxyl radicals from superoxide anions in mitochondria is suggested, as shown in Fig. 6 .

The superoxide anions are generated in the matrix from the inner membrane of mitochondria, and are quenched to hydrogen peroxide by SOD in the matrix. In fact, Nohl et al. [10] in 1978 found the presence of hydrogen peroxide in mitochondria with fluorescence spectroscopy. Most of the hydrogen peroxide migrates through the inner membrane into the membrane space. The hydrogen peroxide changes to hydroxyl radicals by metal-mediated Fenton reaction. On the other hand, one portion of the superoxide anion radicals released into the membrane space must react directly with the metal, for reducing ferric ion to ferrous, to recycle the Fenton reaction.

\section{SUMMARY}

The hydroxyl radicals are known to be a biologically active oxygen species. A remarkable enhancement of hydroxyl radical generation was observed in the mitochondria obtained from ischemic myocardium. The hydroxyl radicals are the primary reactive species leading to cellular damages, such as membrane damages, DNA damages, enzyme inactivation, protein denaturation and so forth. The origin of the hydroxyl radicals appeared in mitochondria was discussed. 
Fig 1

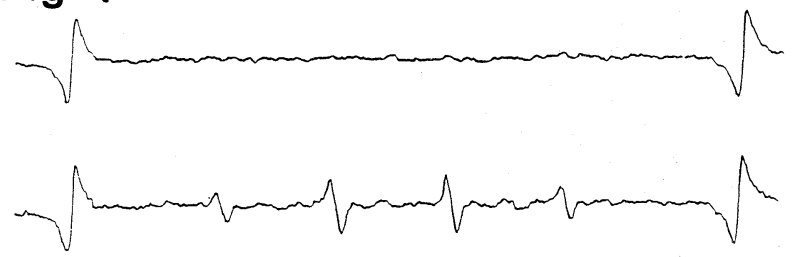

Fig. 1. ESR spectra of DMPO spin adduct obtained from mitochondria.

upper spectrum: succinate alone, lower spectrum: succinate, antimycin A

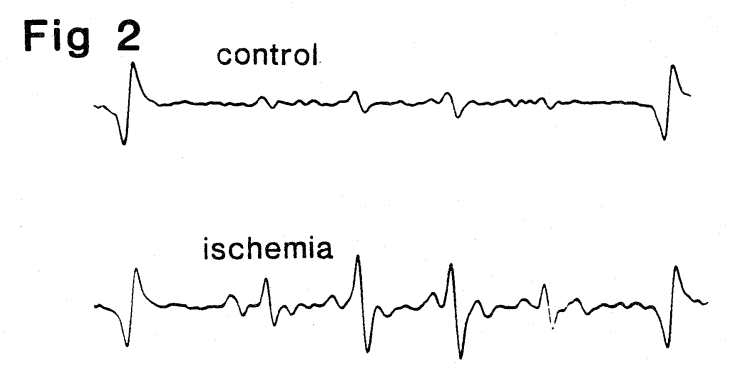

Fig. 2. ESR spectra of DMPO spin adduct obtained from mitochondria in the presence of succinate and antimycin $A$.
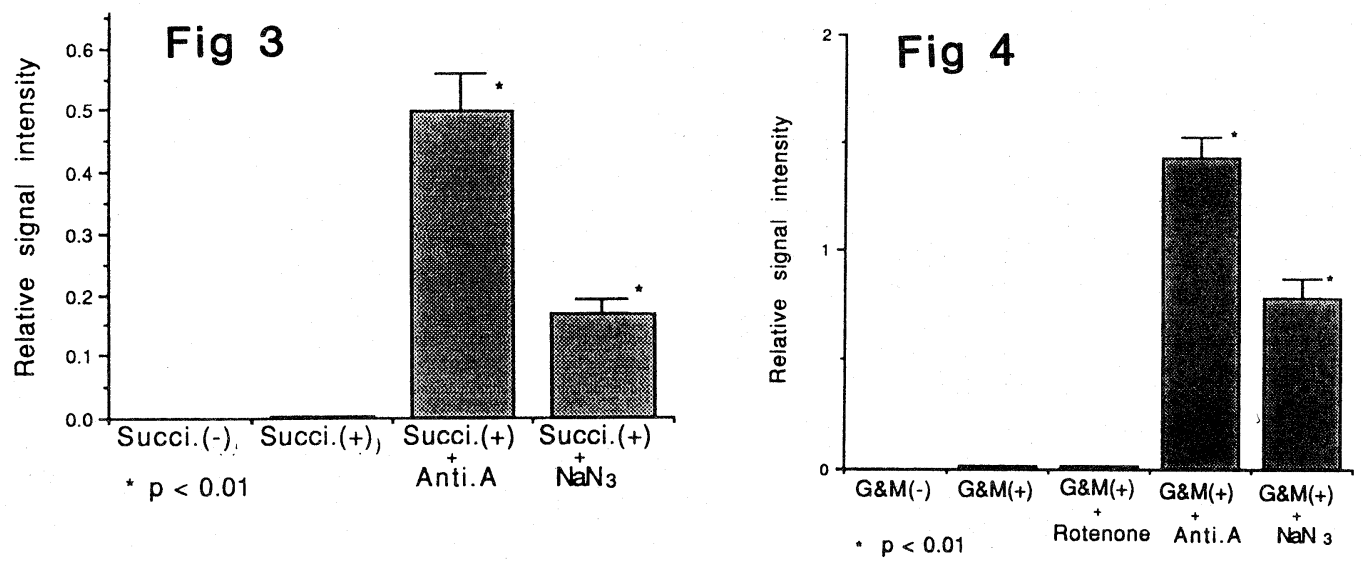

Fig. 3. Relative signal intensity for DMPO-hydroxyl radical adduct obtained from mitochondria respiring with succinate in the presence of electron transfer inhibitor.

Fig. 4. Relative signal intensity for DMPO-hydroxyl radical adduct obtained from mitochondria respiring with glutamate \& malate in the presence of electron transfer inhibitor. 


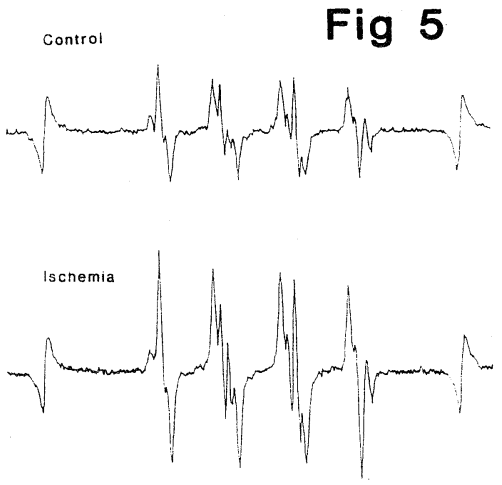

Fig. 5. ESR spectra of DMPO spin adduct obtained from submitochondrial particles.

Fig. 6. Schematic representation of hydroxyl radical generation from mitochondria.

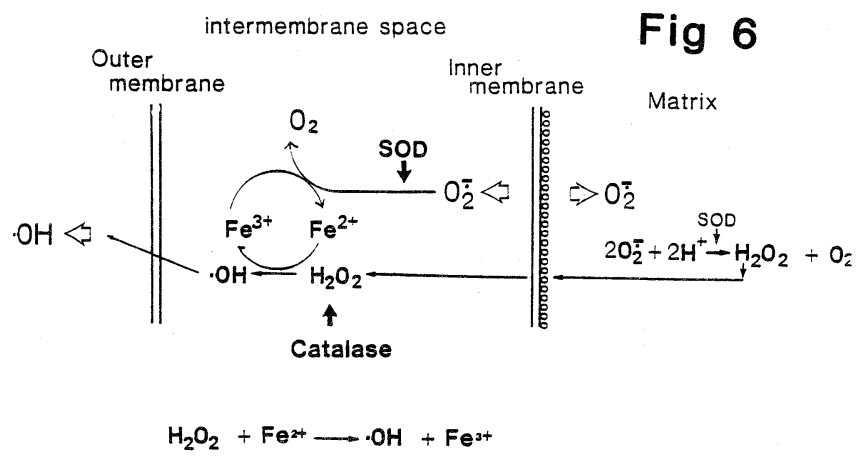

\section{REFERENCES}

[1] McCord, J.M. (1988): Oxygen-derived free radicals in post-ischemic tissue injury. New Engl. J. Med., 312, 159-163.

[2] Flaherty, J.M., and Weisfeldt, M.L. (1988): Respiration injury. Free Rad. Biol. \& Med., 5, 409-419.

[3] Zweier, J.L. (1988): Measurement of superoxide-derived free radicals in the reperfused heart. J. Biol. Chem., 263, 1353-1357.

[4] Nohl, H., Jordan, W., and Hegner, D. (1981): Identification of free hydroxyl radicals in respiring rat heart mitochondria by spin trapping with the nitrone DMPO. FEBS Lett., 123, 241-244.

[5] Shin, G., Sugiyama, M., Shoji, T., Kagiyama, A., Sato, H., and Ogura, R. (1989): Detection of mitochondrial damages in myocardial ischemia with ESR spin labeling technique. J. Mol. Cell Cardiol., 21, 1029-1036.

[6] Ueta, H., Ogura, R., Sugiyama, M., Kagiyama, A., and Shin, G. (1990): Ospin trapping on cardiac submitochondrial particles isolated from ischemic and non-ischemic myocardium. J. Mol. Cell Cariol., 22, 893-899.

[7] Ogura, R., Sugiyama, M., Haramaki, N., and Hidaka, T. (1991): Electron spin resonance studies on the mechanism of adriamycin-induced heart mitochondrial damages. Cancer Res., 51, 3555-3558.

[8] Nishi, J., Ogura, R., Sugiyama, M., Hidaka, T., and Kohno, M. (1991): Involvement of active oxygen in lipid peroxide radical reaction of epidermal homogenate following ultraviolet light exposure. J. Invest. Dermat., 97, 115-119.

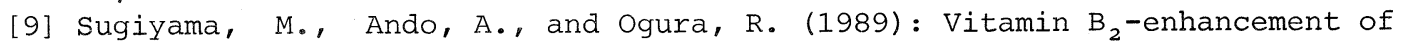
sodium chromate (VI)-induced DNA single strand breaks: ESR study of the action of vitamin $\mathrm{B}_{2}$. Biochem. Biophys. Res. Commun., 159, 1080-1085.

[10] Nohl, H., and Hegner, D. (1978): Do mitochondria produce oxygen free radicals in vivo? Eur. J. Biochem., 8, 563-567. 